\title{
P01-10-7 Poster session
}

\section{Type 2 Diabetes Mellitus patients with comorbidities in a South African managed healthcare organization}

\author{
Lovina A Naidoo', Neil Butkow ${ }^{1}$, Paula Barnard ${ }^{2}$, Elena Libhaber ${ }^{3}$ \\ ${ }^{I}$ Pharmacy and Pharmacology, Faculty of Health Sciences, University of Witwatersrand, Johannesburg, South Africa, \\ ${ }^{2}$ School of Therapeutic Sciences, Faculty of Health Sciences, University of Witwatersrand, Johannesburg, South Africa, \\ ${ }^{3}$ School of Clinical Medicine, Faculty of Health Sciences, University of Witwatersrand, Johannesburg, South Africa
}

Background:

Approximately $80 \%$ of Type 2 Diabetes Mellitus (T2DM) patients have at least one additional condition creating additional pressure on the healthcare system. In the managed healthcare arena in South Africa chronic diseases such as T2DM and Major Depressive Disorder (MDD) are seen as discrete and are managed independently. In patients with coronary artery disease, identification and treatment of MDD has shown a $42 \%$ reduction in death or recurrent myocardial infarction. T2DM patients have an inherently higher cardiovascular risk profile which may suggest a bidirectional relationship between T2DM and MDD. The objective was to examine the hospital admission and length of stay in T2DM and MDD patients within a managed healthcare organization.

Methods:

This study is a retrospective review of claims data of registered T2DM patients at a managed healthcare organization in South Africa for the year 2014. In T2DM patients, comorbidities were defined as concordant such as cardiovascular diseases or discordant i.e. Cancer and MDD. Hospital resource utilization was extracted by number of annual admissions, length of stay and average cost per admission.

Results:

Among the 902 registered T2DM patients within the healthcare organization 153 (17\%) were identified with having MDD. The T2DM group without MDD was predominantly male (62\%) and 58 years old. The group with T2DM and MDD was mainly female (64\%) and slightly older at 60 years of age. The T2DM + MDD group revealed an 18\% hospital admission rate compared to the T2DM - MDD patients (10\%). Hospital patterns of the T2DM and MDD patients are shown in Table 1.

Conclusions:

Significantly more patients with MDD and T2DM were hospitalized. There was no difference in the average number of admissions and length of stay in hospital between the T2DM patients with and those without MDD. Preliminary analysis shows no cost difference between the hospitalized T2DM patients with and without MDD. Given the relative incidence of MDD future healthcare initiatives should be aimed at recognizing underlying MDD in patients with T2DM. Healthcare organizations need to focus on combined integrated multiple disease management in T2DM and MDD patients to lower the financial burden and improve outcomes. 